\title{
169 Transitional cell papilloma (Ringertz tumour)
}

A Is usually indistinguishable from an allergic nasal polyp.

B Most arise from the nasal septum.

C May have areas of columnar and squamous epithelium.

D Malignant transformation only occurs after irradiation.

$\mathrm{E}$ Complete removal is the treatment of choice.

\section{Benign tumours of the paranasal sinuses}

A Ivory osteoma of the frontal sinus is the commonest type.

B The commonest presentation is headache due to pressure erosion of the anterior cranial fossa.

C Mucocoele and ocular displacement are complications of frontal sinus osteoma.

D Localized fibro-osseous dysplasia in children should be excised completely because of the danger of malignant change.

E Hyperostosis frontalis interna is a cause of headache in elderly females.

\section{Malignant tumours of the nose and paranasal sinuses}

A Squamous cell carcinoma is the commonest type.

B Retropharyngeal and cervical lymph node metastases are common.

C Adenoid cystic carcinoma is associated with woodworkers in the furniture industry.

D An ameloblastoma is a rapidly growing, highly malignant tumour.

E Burkitt's lymphoma is confined to African children. 